\title{
Simulation Analysis of the Evolution of Sustainable Operation of Transport Infrastructure Projects under Government Regulation Based on Prospect Theory and BP Neural Network
}

\author{
Chongsen Ma $\mathbb{D}$, Yun Chen $\mathbb{D}$, and Yinghui Zhang $\mathbb{i}$ \\ College of Transportation Engineering, Changsha University of Science and Technology, Hu Nan 410000, China \\ Correspondence should be addressed to Chongsen Ma; machongsen@stu.csust.edu.cn
}

Received 26 September 2021; Revised 12 October 2021; Accepted 19 October 2021; Published 3 November 2021

Academic Editor: Bai Yuan Ding

Copyright ( 2021 Chongsen Ma et al. This is an open access article distributed under the Creative Commons Attribution License, which permits unrestricted use, distribution, and reproduction in any medium, provided the original work is properly cited.

\begin{abstract}
The decisions and actions of operators in the operation of transport infrastructure play a crucial role in the sustainability of the project but are influenced by factors such as the strength of government regulation and the frequency of use by users. The influence of social recognition and acceptance by the authorities on the decisions of the various parties involved in a project is becoming increasingly significant. To address this issue, this paper attempts to apply prospect theory to characterize the impact of changes in recognition on the decisions of project managers and the government from the perspective of recognition and to construct a tripartite evolutionary game model for the government, the operator, and the users, taking into account the combined effect of multiple factors, to explore the evolutionary law of the operator's strategy choice. Evolutionary game theory, in which each person is considered irrational and behavior is changeable, is more realistic. The addition of prospect theory allows the model to more realistically reflect the decisions of each participant in the game process when faced with risk. The results of the study show that there is an optimal level of external regulation to maximize the benefits for all three parties in the game, strong government regulation does not necessarily improve service quality, operators tend to provide low-quality services in the game process and incentives should be increased, and that attempts should be made to provide users with a variety of transport infrastructure options to ensure that users' interests are maximized. The paper further analyzes the indicators established by sensitive factors using BP neural networks on the basis of the analysis of transportation infrastructure operation and impact sensitive factors using evolutionary games and finds that the conclusions obtained by deep learning algorithms are more consistent with those obtained using evolutionary games, achieving cross-validation of the results. The reliability of the result is improved, and it is demonstrated that deep learning algorithms can be introduced as a supplement in the process of future analysis of transportation infrastructure operations. Finally, management suggestions are made in light of the actual situation.
\end{abstract}

\section{Introduction}

As an important part of urban infrastructure, transport infrastructure has a large impact on the development and economic growth of cities. How to achieve efficient transport infrastructure operations and guarantee the sustainability of the project during its life cycle is a problem that the government must face [1]. Besley and Ghatak studied how to allocate the public and private sector controllers for purely public goods [2]. At the same time, due to the existence of uncertainty in the project operation process and the incompleteness in the agreement, the project may generate risks, and the government must prevent the operator from damaging the public interest through certain means to prevent the operator from damaging the public interest [3]. Some scholars believe that risks should be avoided through supplementary mechanisms [4]. Through the above-mentioned scholars' studies, it can be found that, in the operation process, the interests of the various parties involved in the project are not consistent, and they may damage the overall interests for their own benefit. Therefore, it is of practical significance to study the behavior of the project parties in the operation process and the reasons for their occurrence.

At the level of operational regulation of projects, Xia and Gao argue that as social regulation gradually becomes mainstream, a credit will become the core of social club 
regulation of infrastructure operations [5]. Jiang argues that the main problems of China's infrastructure currently focus on government departments' negligence in supervision, and operators usually choose unreasonable pricing and negligent management strategies in order to expand their interests [6]. He argues that the lack of government policies, regulatory systems, and reasonable risk allocation systems at the regulatory level leads to project risks [7]. El-Gohary et al. argue that when the private sector only cares about its own interests, the probability of adopting a breach of trust strategy to operate rises [8]. The above-mentioned studies show that there are many problems in the process of infrastructure operation and it is difficult to achieve sustainable operation of projects. The lack of government regulation and the private sector's focus on its own interests usually result in reduced benefits for users and the government. The parties involved in the project are unable to maximize the overall benefits.

To address the difficulty of achieving sustainability in the operation of transport infrastructure, $\mathrm{Wu}$ argues that the PPP model should be introduced for the operation of stock assets to expand the sources of financing to revitalize the project [9]; Sun proposes promoting "hybridization" to enhance the benefits of the project through the analysis of two typical projects [10]; Xia et al. used an evolutionary game approach to analyze the impact of different levels of social regulation on the behavior of government departments and the private sector.

However, in the course of many scholars' research, the following two main problems exist.

(1) All parties involved in the project are imperfectly rational in the process of participating in the decision-making game, and they do not precisely perceive the risks of the project and the risks of their behavior, and their perception of risks is influenced by the environment they are in. However, there are relatively few papers in the existing literature that address this point.

(2) Although the existing studies take a variety of approaches, they usually only consider the game between the government and the operator in the analysis process and rarely include users in the study [11-15]. Although transport infrastructure is a quasipublic benefit facility, in the actual operation process, if only government subsidies are considered in the benefit calculation process, without considering the impact of users and social recognition on the project decision in the operation process, it cannot fully meet the actual situation; at the same time, in the current research, less psychological factors such as recognition are included in consideration of the dynamic game, and it is more practical to include recognition in the model [16-20].

(3) In the traditional analysis, the operation of transportation infrastructure is mostly analyzed in the form of game, regression, or expert scoring [21-25]. In the research process, machine learning and deep learning $[26,27]$, which are more studied in recent years, are less involved, and there is less research on whether such methods can be used to predict the operation effect of transportation infrastructure.

The innovation points of this paper are mainly reflected in the following two points:

(1) Based on prospect theory and imperfectly rational decision-making in the study of sustainable operation of transport infrastructure projects under government regulation, it more realistically reflects the choices of the parties involved in the project when facing different degrees of risk.

(2) The modeling process takes into account the impact of behavior on social acceptance and the acceptance of higher regulatory authorities and quantifies it. More realistic and appropriate recommendations are made in response to the simulation results.

(3) The paper uses BP neural networks to predict the operation of transportation infrastructure and finds that the conclusions obtained are closer to those of the traditional evolutionary game approach, which can be better implemented with the traditional approach and facilitate the operation of transportation infrastructure.

\section{Simulation Model Construction}

2.1. Simulation Analysis Model Construction for Sustainable Operation Evolution of Transportation Infrastructure Projects. In recent years, transport infrastructure projects have become more difficult to operate sustainably due to their large scale of construction, high capital investment, and long payback period. However, regardless of the mode of operation, the main interest groups involved can be summarized as follows: the government, the construction operator, and the user. From an economic point of view, the three parties have a mutual influence on each other. The government commissions the developer to build or operate the project, supervises the project with preagreed contracts, and monitors the performance of the project in terms of actual usage; the developer provides services to the users through construction and operation, and the users' satisfaction and frequency of use influence the government's decisions on similar projects. Due to the asymmetry of information in the whole process and the imperfect rationality of the stakeholders in the decision-making process, the construction and operation parties may be driven by their own technical and information advantages to act opportunistically, causing the government to pay more costs and the users to reap higher profits without receiving the services they deserve. To ensure that transport infrastructure projects meet their construction objectives and achieve sustainable operation, the government and user groups should monitor the behavior of the construction operators. The relationship between the main stakeholders in the sustainable operation of transport infrastructure is shown in Figure 1. 


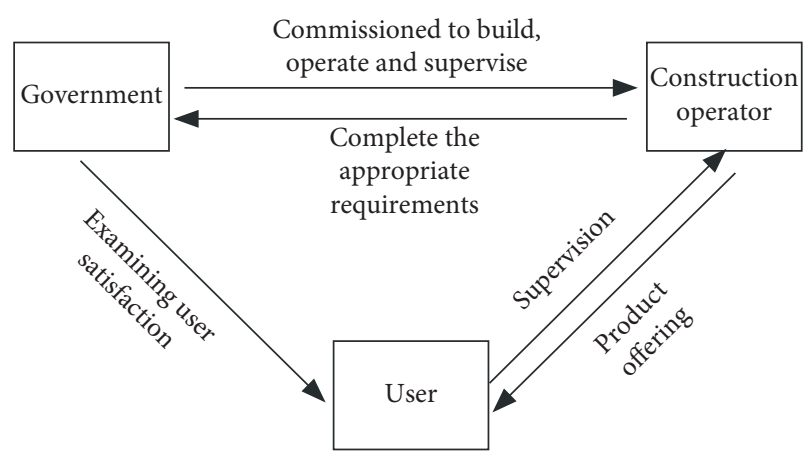

FIgURE 1: Tripartite relationship map of the game.

\subsection{Model Assumptions}

Assumption 1. In transport infrastructure, the government entrusts the operation of the project to the construction operator. The government regularly reviews the construction and operational effectiveness of the project and adjusts the operation subsidy according to the operational effectiveness to ensure that the social benefits of the project are maximized. The construction operator is responsible for the operation of the project and receives a return through user fees and operating subsidies.

Assumption 2. In the operation of transport infrastructure, the government, the construction operator, and the user are all effectively rational "economic agents;" i.e., all parties involved in the project are not fully rational. In the game process, the three parties play a finite number of repeated games.

Assumption 3. Evolutionary games are based on expected utility theory and do not take into account the influence of psychological perception factors on the decision outcome of the game subjects during the decision-making process. In this paper, we use the prospect theory proposed by Kahnerman et al. to correct for the inconsistent behavior of risk preferences of decision subjects. Prospect theory states that one cannot perceive losses and gains in absolute terms but rather the relative value of perceived losses. This value is expressed using $\Delta \omega_{1}$, which is the difference between the actual loss or gain $\omega_{1}$ and a reference point $\omega_{0}$. This reference point is subject to the subjective influence of the decision-maker. The choice of reference point varies in different fields of study. In the field of finance and investment, the average return is mostly chosen as the reference point, whereas in the literature on regulatory research using prospect theory, 0 is mostly chosen as the reference point. In this paper, 0 is chosen as the reference point. In prospect theory, the expected total utility of a decision is measured using a value function $v\left(\Delta \omega_{1}\right)$ and a weight function $\pi$. The prospect value is

$$
V=\sum_{t} \pi(p l) v(\Delta \omega \iota) .
$$

Each participating subject in the game makes a judgment on its next move based on its perceived value of the lost gain, where a value function is

$$
V\left(\Delta \omega_{i}\right)=\left\{\begin{array}{l}
\left(\Delta \omega_{i}\right)^{\theta}, \quad \Delta \omega_{i} \geq 0, \\
-\lambda\left(-\Delta \omega_{i}\right)^{\theta}, \quad \Delta \omega_{i}<0,
\end{array}\right.
$$

where $\theta$ is the risk attitude coefficient, indicating the marginal degree of diminishing perceived value of profit and loss by the game subject; $\lambda$ is the loss avoidance coefficient, indicating the sensitivity of the game subject to loss; the larger the value, the greater the sensitivity of the game subject to loss. At the same time, the game subject judges the weights according to the actual situation of the event, and the judgment formula is

$$
\pi\left(p_{i}\right)=\frac{p^{\gamma}}{\left(p^{\gamma}+(1-p)^{\gamma}\right)^{1 / \gamma}},
$$

with the exception of very small probability events, $\pi\left(p_{i}\right)<p_{i}, \pi\left(p_{i}\right)+\pi\left(1-p_{i}\right) \leq 1$ and $\pi(1)=1, \pi(0)=0$. In prospect theory, the probability of a low-probability event occurring is usually overestimated, and the probability of a high-probability event occurring is usually underestimated.

Assumption 4. The government has two strategic choices based on its own and society's overall benefits: a strong control model and a weak control model. The building operator has two strategies: to provide high-quality services and to provide low-quality services. Users have two strategic choices: high frequency of use and low frequency of use. The probability of the government, the construction operator, and the user choosing the strong control model and providing high-quality services and high frequency of use are $x$, $y, z,(0 \leq x \leq 1,0 \leq y \leq 1,0 \leq z \leq 1)$, respectively; then, the probability of choosing the weak control model and providing low-quality services and low frequency of use is $1-x$, $1-y, 1-z$.

Assumption 5. The government's choice of the "strong control model" means that the government will take appropriate measures to correct any problems that arise during the construction and operation of the project and will make real-time adjustments to the project's subsidies if the operational performance is not up to standard. The "weak control model" means that the government will not adjust the incentives and subsidies according to the operation of the enterprise and will provide a uniform standard subsidy. The construction operator chooses to "provide high-quality services," which means that the operator, in the course of operation, strictly abides by the contractual agreement and gives full play to the initiative of the enterprise, providing services that meet or exceed the requirements of the government and the needs of the applicable parties. To "provide low-quality services" means that the operator has chosen to partially or fully violate the contractual agreement and in some cases has even colluded with government officials. 
2.3. Parameter Setting and Model Construction. If the government chooses the "strong control model," it incurs additional regulatory costs $C_{3}$ and receives incentives from higher authorities and social benefits $\mathrm{SR}_{1}$; if the government chooses the "weak control model," it incurs no additional regulatory costs and receives no additional benefits. If the government chooses the "weak control model," there are no additional regulatory costs and no additional benefits. However, when the operator chooses to "provide a lowquality service," it will be challenged by society, resulting in a combined loss of SR2 (SR2 < SR1, SR1 > C3).

Assuming a fixed revenue of $V$ to be realized by the building operator, an additional cost of $C_{1}$ and an incentive subsidy of $B$ are incurred by the operator if it chooses to "provide a high-quality service." The cost of choosing to "provide a low-quality service" is $C_{2}$ (including loss of goodwill, loss of potential customers, etc.), $C_{3}<C_{1}<C_{2}$, but if the operator chooses to provide a low-quality service, it will incur a penalty of $F$ in the event of government regulation.

There is no additional gain for the user when the operator "provides a high-quality service," regardless of which strategy is chosen. In the case of low-quality services, there is an additional loss of $Y_{1}$ if "high usage" is chosen, and a loss of $Y_{2}$ if "low usage" is chosen. If the government adopts a "strong control model" when the operator is providing a "low-quality service," it will gain an additional $\mathrm{SR}_{3}$ due to the increased trust in the government as a result of the increased government control over the project $\left(\mathrm{SR}_{1}>\mathrm{SR}_{3}, Y_{2}>Y_{1}\right)$.

It is assumed that the probability of the operator being detected by the government for "providing low-quality services" is $\alpha$ under the "weak control model" and $100 \%$ when the government adopts a strong control model.

In this paper, the cost of government regulation and the operating cost of the builder are assumed to be deterministic, while the rest of the parameters are related to subjective perceptions and are calculated using prospective values. The benefit matrix of the evolutionary game for the operation of transport infrastructure projects is shown in Table 1.
2.4. Model Solution. According to Table 1, the prospective and average expectations of the government's "strong control model" and "weak control model" strategies are

$$
\begin{aligned}
E_{11}= & y z *\left(-C_{3}+V\left(\mathrm{SR}_{1}\right)\right)+y(1-z) *\left(-C_{3}+V\left(\mathrm{SR}_{1}\right)\right) \\
& +(1-y) z *\left(-C_{3}+V\left(\mathrm{SR}_{1}\right)+V\left(\mathrm{SR}_{3}\right)\right) \\
& +(1-y) *(1-z) *\left(-C_{3}+V\left(\mathrm{SR}_{1}\right)+V\left(\mathrm{SR}_{3}\right)\right), \\
E_{12}= & y z * 0+y(1-z) * 0+(1-y) z \\
& *\left(-V\left(\mathrm{SR}_{2}\right)\right)+(1-y) *(1-z) *\left(-V\left(\mathrm{SR}_{2}\right)\right), \\
\bar{E}_{13}= & x E_{11}-(1-x) E_{12} .
\end{aligned}
$$

The prospective expectations and mean expectations for the "high-quality service" and "low-quality service" strategies of the operating service providers are

$$
\begin{aligned}
E_{21}= & x z *\left(V-C_{1}+B\right)+x(1-z) *\left(V-C_{1}+B\right) \\
& +(1-x) z *\left(V-C_{1}+B\right)+(1-x) *(1-z) *\left(V-C_{1}+B\right), \\
E_{22}= & x z *\left(V-C_{2}-V(F)\right)+x(1-z) *\left(V-C_{2}-V(F)\right) \\
& +(1-x) z *\left(V-C_{2}-V(F)\right)+(1-x) *(1-z) \\
& *\left(V-C_{2}-V(F)\right), \\
\bar{E}_{23}= & y E_{21}-(1-y) E_{22} .
\end{aligned}
$$

The prospective expectations and mean expectations of users adopting the "high use" and "low use" strategies are

$$
\begin{aligned}
E_{31}= & x y * 0+x(1-y) *-\left(V\left(Y_{1}\right)\right)+(1-x) y * 0 \\
& +(1-x) *(1-y) *-\left(V\left(Y_{1}\right)\right), \\
E_{32}= & x y * 0+x(1-y) *-\left(V\left(Y_{2}\right)\right)+(1-x) y * 0 \\
& +(1-x) *(1-y) *\left(-V\left(Y_{2}\right)\right), \\
\bar{E}_{33}= & z E_{31}-(1-z) E_{32} .
\end{aligned}
$$

The replicated dynamic differential equation for the government side, the building operator, and the user side choosing an active strategy can be expressed:

$$
\begin{aligned}
F(x)= & \frac{\mathrm{d} x}{\mathrm{~d} t}=x *\left(E_{11}-\bar{E}_{13}\right)=x *(1-x) *\left(y z * V\left(\mathrm{SR}_{1}\right)-C_{3}\right)+y *(1-z) *\left(V\left(\mathrm{SR}_{1}-C_{3}\right)\right)+(1-y) * z \\
& *\left(V\left(\mathrm{SR}_{1}\right)+V\left(\mathrm{SR}_{2}\right)+V\left(\mathrm{SR}_{3}\right)-C_{3}\right)+(1-y) *(1-z) *\left(V\left(\mathrm{SR}_{1}\right)+V\left(\mathrm{SR}_{2}\right)+V\left(\mathrm{SR}_{3}\right)\right)-C_{3}, \\
F(y)= & \frac{\mathrm{d} y}{\mathrm{~d} t}=y *\left(E_{21}-\bar{E}_{23}\right)=y *(1-y) *\left(x z *\left(B-C_{1}+C_{2}+V\right)(F)\right)+x *(1-z) *\left(B-C_{1}+C_{2}+V\right)(F) \\
& +(1-x) * z *\left(B-C_{1}+C_{2}+\alpha\right) V(F)+(1-x) *(1-z) *\left(B-C_{1}+C_{2}+\alpha V(F)\right), \\
F(z)= & \frac{\mathrm{d} z}{\mathrm{~d} t}=z *(1-z) *(x *(1-y)) *\left(V\left(Y_{2}\right)-V\left(Y_{1}\right)\right)+(1-x) *(1-y) *\left(V\left(Y_{2}\right)-V\left(Y_{1}\right)\right) .
\end{aligned}
$$


TABLE 1: Revenue matrix.

\begin{tabular}{|c|c|c|c|c|c|}
\hline \multirow{3}{*}{ Strategy } & & \multicolumn{4}{|c|}{ Government } \\
\hline & & \multicolumn{2}{|c|}{ Strong control model } & \multicolumn{2}{|c|}{ Weak control model } \\
\hline & & High-quality service & Low-quality services & High-quality service & Low-quality services \\
\hline User & High usage & $\begin{array}{c}-C_{3}+V\left(\mathrm{SR}_{1}\right) \\
V-C_{1}+B \\
0 \\
-C_{3}+V\left(\mathrm{SR}_{1}\right) \\
V-C_{1}+B \\
0 \\
\end{array}$ & $\begin{array}{c}-C_{3}+V\left(\mathrm{SR}_{1}\right)+V\left(\mathrm{SR}_{3}\right) \\
V-C_{2}-V(F) \\
-V\left(Y_{1}\right) \\
-C_{3}+V\left(\mathrm{SR}_{1}\right)+V\left(\mathrm{SR}_{3}\right) \\
V-C_{2}-V(F) \\
-V\left(Y_{2}\right)\end{array}$ & $\begin{array}{c}0 \\
V-C_{1}+B \\
0 \\
0 \\
V-C_{1}+B \\
0 \\
\end{array}$ & $\begin{array}{c}-V\left(\mathrm{SR}_{2}\right) \\
V-C_{2}-\alpha V(F) \\
-V\left(Y_{1}\right) \\
-V\left(\mathrm{SR}_{2}\right) \\
V-C_{2}-\alpha V(F) \\
-V\left(Y_{2}\right) \\
\end{array}$ \\
\hline
\end{tabular}

When the probability of the government side choosing the strong control model is 1 , the government gains an additional $\mathrm{SR}_{1}$; when the government side chooses the weak control model, the government gains an additional 0 . The prospective value of SR1 is

$$
\begin{aligned}
V\left(\mathrm{SR}_{1}\right) & =\pi(1) * V\left(\mathrm{SR}_{1}\right) \\
& =\left(\mathrm{SR}_{1}\right)^{\theta} .
\end{aligned}
$$

When the probability of the construction operator choosing a low-quality service is 1 , the penalty imposed by the higher level of government is $F$; when a high-quality service is chosen, the penalty received is 0 . The prospective value of $F$ is

$$
\begin{aligned}
V(-F) & =\pi(1) *(-F) \\
& =-\lambda F^{\theta} .
\end{aligned}
$$

When the probability of a user taking a high frequency of use is 1 , the operator gains a loss of $Y_{1}$ if it provides a lowquality service; when a low frequency of use is chosen, the loss is reduced to $Y_{2}$; the prospect value is

$$
\begin{aligned}
V\left(Y_{1}\right) & =\pi(1) * V\left(-Y_{1}\right) \\
& =-\lambda Y_{1}^{\theta}, \\
V\left(Y_{2}\right) & =\pi(1) * V\left(-Y_{2}\right) \\
& =-\lambda Y_{2}^{\theta} .
\end{aligned}
$$

Similarly, the prospect values of $\mathrm{SR}_{2}$ and $\mathrm{SR}_{3}$ can be obtained as

$$
\begin{aligned}
V\left(\mathrm{SR}_{2}\right) & =\pi(1) * V\left(\mathrm{SR}_{2}\right) \\
& =-\lambda\left(\mathrm{SR}_{2}\right)^{\theta}, \\
V\left(\mathrm{SR}_{3}\right) & =\pi(1) * V\left(\mathrm{SR}_{3}\right) \\
& =\left(\mathrm{SR}_{3}\right)^{\theta} .
\end{aligned}
$$

Substituting the prospect values yields a dynamic equation for the replication of the active strategy of the three-way game.

$$
\begin{aligned}
& F(x)=x *(1-x) *\left(y z^{*}\right) *\left(\left(\mathrm{SR}_{1}\right)^{\theta}-C_{3}\right) \\
& +y *(1-z) *\left(\left(\mathrm{SR}_{1}\right)^{\theta}-\mathrm{C}_{3}\right)+(1-y) * z * \\
& +\left(\left(\mathrm{SR}_{1}\right)^{\theta}-\lambda\left(\mathrm{SR}_{2}\right)^{\theta}+\left(\mathrm{SR}_{3}\right)^{\theta}-\mathrm{C}_{3}\right)+(1-y) \\
& *(1-z) *+\left(\left(\mathrm{SR}_{1}\right)^{\theta}-\lambda\left(\mathrm{SR}_{2}\right)^{\theta}+\left(\mathrm{SR}_{3}\right)^{\theta}-C_{3}\right) \text {, } \\
& F(y)=y *(1-y) *\left(x z *\left(B-C_{1}+C_{2}-\lambda F^{\theta}\right)\right)+x *(1-z) \\
& *\left(B-C_{1}+C_{2}-\lambda F^{\theta}\right)+(1-x) * z *\left(B-C_{1}+C_{2}-\alpha \lambda F^{\theta}\right) \\
& +(1-x) *(1-z) *\left(B-C_{1}+C_{2}-\alpha \lambda F^{\theta}\right) \text {, } \\
& \begin{aligned}
F(z)= & z *(1-z) *(x *(1-y)) *\left(\lambda Y_{1}^{\theta}-\lambda Y_{2}^{\theta}\right) \\
& +(1-x) *(1-y) *\left(\lambda Y_{1}^{\theta}-\lambda Y_{2}^{\theta}\right)
\end{aligned}
\end{aligned}
$$

\section{Equilibrium Analysis of the Tripartite Game for the Operation of Transport Infrastructure Projects}

\subsection{Unilateral Stabilization Strategies for Game Subjects}

3.1.1. Government-Side Progressive Stability Analysis. Let equation (12) equal 0 and solve for $x=0, x=1, y=\lambda\left(\mathrm{SR}_{2}\right)$ $\theta_{-}-\left(\mathrm{SR}_{3}\right)^{\theta}-\left(\mathrm{SR}_{1}\right)^{\theta}+C_{3} / \lambda\left(\mathrm{SR}_{2}\right)^{\theta}-\left(\mathrm{SR}_{3}\right)^{\theta}-2 Z C_{3}=Y^{*}$. It follows from the stability theorem for replicating dynamic differential equations that $F(x)=0$, and $\partial F(x) / \partial x<0$, $\mathrm{x}$ is an evolutionary stabilization strategy.

When $y=Y^{*}, F(x)=0$ constantly established. The stability point is $x=0, x=1$. Any value of $x$ is a steady state; i.e., the strategy of the construction unit does not change over time.

When $y<Y^{*}, F(x)=0$ constantly established. And $\left\{\begin{array}{l}\partial F(x) / \partial x>0, x=0 \\ \partial F(x) / \partial x<0, x=1\end{array}\right.$ established. The stability point is $x=1$. Any value of $x$ is a steady state. That is, this suggests that the perceived benefits of strong control measures are greater than the costs of regulation. In this regard, the benefits include multiple gains, and it is clear from prospect theory that game players are usually reluctant to take losses when faced with 
gains; i.e., government departments usually tend to choose the strong control model to avoid incurring losses.

When $y>Y^{*}, F(x)=0$ constantly established. And $\left\{\begin{array}{l}\partial F(x) / \partial x>0, x=1 \\ \partial F(x) / \partial x<0, x=0\end{array}\right.$ established. The stability point is $x=0$. Any value of $x$ is a steady state. That is, it shows that government departments are more sensitive to the perceived cost of inputs than the returns they receive when taking strong control measures. They prefer to bear penalties with uncertainty rather than invest more in regulatory costs. That is, the government tends to choose a weak control model. In this case, the higher level of government should take measures to assign responsibility and increase control, making the government strengthen its control over the project.

3.2. Construction Operator Progressive Stability Analysis. Let equation (13) equal 0 and solve for $y=0, y=1, z=B-$ $C_{1}+C_{2}-\alpha \lambda F^{\theta}-x \lambda F^{\theta}+\alpha x \lambda F^{\theta} / 2 x\left(B-C_{1}+C_{2}-x \lambda F^{\theta}\right)$

$=Z^{*}$. It follows from the stability theorem for replicating dynamic differential equations that $F(y)=0, \partial F(y) / \partial y<0$, and $x$ is an evolutionary stabilization strategy.

When $z=Z^{*}, F(y)=0$ constantly established. The stability point is $y=0, y=1$. Any value of $y$ is a steady state; i.e., the strategy of the construction unit does not change over time.

When $z<Z^{*}, F(y)=0$ constantly established. And $\left\{\begin{array}{l}\partial F(y) / \partial y>0, y=0 \\ \partial F(y) / \partial y<0, y=1\end{array}\right.$ established. The stability point is $y=1$; Any value of $x$ is a steady state. That is, it shows that the sum of the penalties and losses received by the building operator for providing a low-quality service is greater than the benefits under this strategy. If the building operator adopts a speculative strategy, it will face inevitable losses. In this case, the building operator tends to choose to provide a high-quality service.

When $z>Z^{*}, F(y)=0$ constantly established. And $\left\{\begin{array}{l}\partial F(y) / \partial y>0, x=1 \\ \partial F(y) / \partial y<0, x=0\end{array}\right.$ established. The stability point is $y=0$. It indicates that the penalties and losses received by the building operator in providing low-quality services are less than the gains under this strategy. The building operator tends to choose a speculative strategy to obtain higher returns in this situation.

3.3. User Progressive Stability Analysis. Let equation (14) equal 0 and solve for $z=0, z=1, x=\lambda\left(\mathrm{SR}_{2}\right)^{\theta}-\left(\mathrm{SR}_{3}\right)^{\theta}-$ $\left(\mathrm{SR}_{1}\right)^{\theta}+C_{3} / \lambda\left(\mathrm{SR}_{2}\right)^{\theta}-\left(\mathrm{SR}_{3}\right)^{\theta}-2 Z C_{3}=X^{*}$. It follows from the stability theorem for replicating dynamic differential equations that $F(z)=0, \partial F(z) / \partial z<0$, and $x$ is an evolutionary stabilization strategy.

When $x=X^{*}, F(z)=0$ constantly established. The stability point is $z=0, z=1$. Any value of $z$ is a steady state; i.e., the strategy of the construction unit does not change over time.

When $x<X^{*}, F(z)=0$ constantly established. And $\partial F(z) / \partial z>0, z=0$ $\left\{\begin{array}{l}\partial F(z) / \partial z>0, z=0 \\ \partial F(z) / \partial z<0, z=1\end{array}\right.$ established. The stability point is $z=1$. Any value of $z$ is a steady state. That is, this situation indicates that the user receives more losses than gains when adopting a low frequency of use, in which case the user tends to choose a high frequency of use.

When $x>X^{*}, F(z)=0$ constantly established. And $\left\{\begin{array}{l}\partial F(z) / \partial z>0, z=1 \\ \partial F(z) / \partial z<0, z=0\end{array}\right.$ established. The stability point is $z=0$. This situation indicates that the loss suffered by the user by adopting a low frequency of use is less than the gain, in which case the user tends to choose a low frequency of use.

3.4. Mixed Stabilization Strategy for Gaming Subjects. Let $F(x)=F(y)=F(z)=0$. The equilibrium points can be obtained as follows: $E_{1}=(0,0,0), E_{2}=(1,0,0), E_{3}=(0,1,0)$, $E_{4}=(0,0,1), E_{5}=(1,1,0), E_{6}=(1,0,1), E_{7}=(0,1,1), E_{8}=(1,1,1)$, and $E_{9}=\left(x^{*}, y^{*}, z^{*}\right)$, where $\mathrm{E}_{9}$ is the mixed strategy equilibrium point. If the equilibrium point in the three-way evolutionary game is ESS, it must be satisfied that the equilibrium point is a pure strategy equilibrium, and therefore, only the asymptotic stability of $E_{1}-E_{8}$ needs to be discussed. The asymptotic stability of the system can be obtained from the analysis of the Jacobian matrix, as proposed by Friedman:

$$
J=\left[\begin{array}{lll}
\frac{\partial F(x)}{\partial x} & \frac{\partial F(x)}{\partial y} & \frac{\partial F(x)}{\partial z} \\
\frac{\partial F(y)}{\partial x} & \frac{\partial F(y)}{\partial y} & \frac{\partial F(y)}{\partial z} \\
\frac{\partial F(y)}{\partial x} & \frac{\partial F(y)}{\partial y} & \frac{\partial F(y)}{\partial z}
\end{array}\right]
$$

$$
=\left[\begin{array}{ccc}
{\left[\left(\mathrm{SR}_{2}\right)^{\theta} \lambda(y-1)+(1-y)\left(\mathrm{SR}_{3}\right)^{\theta}+\left(\mathrm{SR}_{1}\right)^{\theta}-\mathrm{C}_{3}\right](1-2 x)} & x(1-x)\left[\lambda\left(\mathrm{SR}_{2}\right)^{\theta}-\left(\mathrm{SR}_{3}\right)^{\theta}\right] & 0 \\
y(1-y) F^{\theta} \lambda(\alpha-1) & {\left[F^{\theta} \alpha \lambda(x-1)-F^{\theta} \lambda x+B-C_{1}+C_{2}\right](1-2 y)} & 0 \\
0 & z(1-z) \lambda\left(Y_{2}{ }^{\theta}-Y_{1}{ }^{\theta}\right) & (1-y) \lambda\left(Y_{1}{ }^{\theta}-Y_{2}{ }^{\theta}\right)(1-2 z)
\end{array}\right] .
$$


Each of the eight equilibrium points is substituted into the Jacobian matrix, and if the eigenvalues of the corresponding matrix are all negative, then the equilibrium point is the ESS of the system, and the eigenvalues of each point are shown in Table 2.

This paper makes the following assumptions, $\left(\mathrm{SR}_{2}\right)^{\theta} \lambda<\left(\mathrm{SR}_{1}\right)^{\theta}<\left(\mathrm{SR}_{3}\right)^{\theta}$. Under prospect theory, the loss to social challenge is less than the reward to government regulation; i.e., the government tends to adopt an active strategy to control the project. The stabilization strategy of the game is analyzed in the following cases:

Situation $\quad 1: \quad\left(\mathrm{SR}_{2}\right)^{\theta} \lambda+C_{3}<\left(\mathrm{SR}_{3}\right)^{\theta}+\left(\mathrm{SR}_{1}\right)^{\theta}$, $B<F^{\theta} \alpha \lambda+C_{1}-C_{2}$. In this scenario, the operator chooses to provide a low-quality service, resulting in a combined loss that is less than the cost of government regulation, while the benefit to the operator of reducing the quality of the service is greater than the government's incentive for high-quality service. From the table, we can find that this is the game model evolution equilibrium point, and evolution equilibrium strategy is strong control mode, low-quality service, and low frequency of use.

Situation $\quad 2: \quad\left(\mathrm{SR}_{2}\right)^{\theta} \lambda+C_{3}>\left(\mathrm{SR}_{3}\right)^{\theta}+\left(\mathrm{SR}_{1}\right)^{\theta}$, $B<F^{\theta} \alpha \lambda+C_{1}-C_{2}$. In this case, the combined loss caused by the operator choosing to provide a lowquality service is greater than the cost of government regulation, while the gain to the operator from reducing the quality of the service is greater than the government's incentive for a high-quality service. Table 3 shows that this is the equilibrium point for the evolution of the game model, and the evolutionary equilibrium strategy is a weak control model, low-quality service, and low frequency of use.

Situation $\quad 3: \quad\left(\mathrm{SR}_{2}\right)^{\theta} \lambda+C_{3}<\left(\mathrm{SR}_{3}\right)^{\theta}+\left(\mathrm{SR}_{1}\right)^{\theta}$, $B>F^{\theta} \alpha \lambda+C_{1}-C_{2}$. In this case, the combined losses incurred by the operator when it chooses to provide a low-quality service are less than the cost of government regulation, while the revenue gained by the operator for reducing the quality of the service is less than the government's incentive for a high-quality service.

Situation $\quad 4: \quad\left(\mathrm{SR}_{2}\right)^{\theta} \lambda+C_{3}>\left(\mathrm{SR}_{3}\right)^{\theta}+\left(\mathrm{SR}_{1}\right)^{\theta}$, $B>F^{\theta} \alpha \lambda+C_{1}-C_{2}$. In this case, the combined losses incurred when an operator chooses to provide a lowquality service are greater than the cost of government regulation, while the revenue gained by the operator for reducing the quality of the service is less than the government's incentive for a high-quality service.

There is no stable equilibrium strategy under situation 3 and situation 4 .

The above analysis shows that, in the actual operation of the project, the operator usually chooses to provide low-quality services out of self-interest, in order to obtain high revenue by the number of operations. Therefore, in the process of gaming the project, the government should set up reasonable penalties to enhance the willingness of the operators to provide highquality services.

\section{Analysis of Simulations}

In order to verify the validity of the evolutionary stability analysis, the model was assigned according to realistic conditions, and numerical simulations were carried out using Python. The parameters set in this paper are shown in Table 4.

Firstly, in order to analyze the impact of the change in the regulatory cost $C_{3}$ on the process and outcome of the evolutionary game, $C_{3}$ is assigned to 10,14 , and 18 , respectively, and the simulation results of replicating the system of dynamic equations evolving 50 times over time are shown in Figure 2. In order to analyze the impact of loss avoidance coefficients on the process and outcome of the evolutionary game, the values of $\mathrm{C}_{3}$ are assigned as 1,2 , and 3 , respectively, and the simulation results are shown in Figure 3.

The graph of the impact of regulatory costs shows that as the cost of regulation rises, it accelerates the rate at which the operating companies and the government side evolve to a point of stability. As the cost of regulation rises, the probability of the government choosing a strong control model decreases. Therefore, when choosing a strong control model, the government needs to be careful to control the cost of control so that it is within a reasonable range. For projects in an unstable state (e.g., in the early stages of operation or within a special period), the regulatory requirements should be appropriately relaxed to facilitate the project to get on track and achieve sustainable operation as soon as possible.

The risk aversion coefficient influence diagram shows that as the risk aversion coefficient rises in the evolutionary process, the probability of the government side choosing a strong control model and the operator choosing a highquality service rises. Therefore, during the operation of the project, priority should be given to risk-sensitive operators, and the penalties for accidents should be increased to raise the risk awareness of operators and increase the probability of operators choosing high-quality services.

The government incentive subsidy B was then analyzed by assigning $B$ values of 6,16 , and 26 and replicating the simulation results for the system of dynamic equations evolving 50 times over time as shown in Figure 4.

The graph of the impact of incentive subsidies shows that as the incentive subsidy rises, it reduces the rate at which the operator and the government side evolve to a stable point. The evolutionary process shows that if the incentive subsidies are increased, to a certain extent, it will reduce the incentive of the operator to improve the service quality. Therefore, the government should formulate a reasonable subsidy incentive policy to avoid the negative effects of high subsidies.

Substituting the parameters so that they evolve 50 times from the initial strategy combination over time, the results are shown in Figures 5-7.

Figures 5-7 show that there is a stable point of evolution of the system (strong control model, low-quality service, and low usage frequency) under the condition that scenario 1 is satisfied. In the case of scenario 2, there is a stable 
TABLE 2: Eigenvalues.

\begin{tabular}{lcrr}
\hline Equalization points & Eigenvalue $\lambda_{1}$ & Eigenvalue $\lambda_{2}$ & Eigenvalue $\lambda_{3}$ \\
\hline$E_{1}(0,0,0)$ & $-\left(\mathrm{SR}_{2}\right)^{\theta} \lambda+\left(\mathrm{SR}_{3}\right)^{\theta}+\left(\mathrm{SR}_{1}\right)^{\theta}-C_{3}$ & $-F^{\theta} \alpha \lambda+B-C_{1}+C_{2}$ & $\lambda\left(Y_{1}^{\theta}-Y_{2}^{\theta}\right)$ \\
$E_{2}(1,0,0)$ & $\left(\mathrm{SR}_{2}\right)^{\theta} \lambda-\left(\mathrm{SR}_{3}\right)^{\theta}-\left(\mathrm{SR}_{1}\right)^{\theta}+C_{3}$ & $-F^{\theta} \alpha \lambda+B-C_{1}+C_{2}$ & $\lambda\left(Y_{1}^{\theta}-Y_{2}^{\theta}\right)$ \\
$E_{3}(0,1,0)$ & 0 & $\left(\mathrm{SR}_{1}\right)^{\theta}-C_{3}$ & $F^{\theta} \alpha \lambda-B+C_{1}-C_{2}$ \\
$E_{4}(0,0,1)$ & $-\left(\mathrm{SR}_{2}\right)^{\theta} \lambda+\left(\mathrm{SR}_{3}\right)^{\theta}+\left(\mathrm{SR}_{1}\right)^{\theta}-C_{3}$ & $\lambda\left(Y_{2}^{\theta}-Y_{1}^{\theta}\right)$ & $-F^{\theta} \alpha \lambda+B-C_{1}+C_{2}$ \\
$E_{5}(1,1,0)$ & 0 & $-\left(\mathrm{SR}_{1}\right)^{\theta}+C_{3}$ & $F^{\theta} \lambda-B+C_{1}-C_{2}$ \\
$E_{6}(1,0,1)$ & $\left(\mathrm{SR}_{2}\right)^{\theta} \lambda-\left(\mathrm{SR}_{3}\right)^{\theta}-\left(\mathrm{SR}_{1}\right)^{\theta}+C_{3}$ & $-F^{\theta} \lambda+B-C_{1}+C_{2}$ & $\lambda\left(Y_{2}^{\theta}-Y_{1}^{\theta}\right)$ \\
$E_{7}(0,1,1)$ & 0 & $\left(\mathrm{SR}_{1}\right)^{\theta}-C_{3}$ & $F^{\theta} \alpha \lambda-B+C_{1}-C_{2}$ \\
$E_{8}(1,1,1)$ & 0 & $-\left(\mathrm{SR}_{1}\right)^{\theta}+C_{3}$ & $F^{\theta} \lambda-B+C_{1}-C_{2}$ \\
\hline
\end{tabular}

TABLE 3: The equilibrium stability of the nonlinear dynamic system for eight situations.

\begin{tabular}{lccccccccccccccccccc}
\hline & \multicolumn{4}{c}{ Situation 1} & \multicolumn{1}{c}{ Situation 2} & \multicolumn{4}{c}{ Situation 3} & \multicolumn{4}{c}{ Situation 4} \\
& $\lambda_{1}$ & $\lambda_{2}$ & $\lambda_{3}$ & Stability & $\lambda_{1}$ & $\lambda_{2}$ & $\lambda_{3}$ & Stability & $\lambda_{1}$ & $\lambda_{2}$ & $\lambda_{3}$ & Stability & $\lambda_{1}$ & $\lambda_{2}$ & $\lambda_{3}$ & Stability \\
\hline$E_{1}(0,0,0)$ & + & - & - & - & - & - & - & ESS & + & + & - & - & - & + & - & - \\
$E_{2}(1,0,0)$ & - & - & - & ESS & + & - & - & - & - & + & - & - & + & + & - & - \\
$E_{3}(0,1,0)$ & 0 & + & + & - & 0 & + & + & - & 0 & + & - & - & 0 & + & & - \\
$E_{4}(0,0,1)$ & + & + & - & - & - & + & - & - & + & + & + & - & - & + & + & - \\
$E_{5}(1,1,0)$ & 0 & - & + & - & 0 & - & + & - & 0 & - & - & - & 0 & - & - & - \\
$E_{6}(1,0,1)$ & - & - & + & - & + & - & + & - & - & + & + & - & + & + & + & - \\
$E_{7}(0,1,1)$ & 0 & + & + & - & 0 & + & + & - & 0 & + & - & - & 0 & + & - & - \\
$E_{8}(1,1,1)$ & 0 & - & + & - & 0 & - & + & - & 0 & - & - & - & 0 & - & - & - \\
\hline
\end{tabular}

TABle 4: Assignment table.

\begin{tabular}{lc}
\hline Parameters & Assignment \\
\hline $\mathrm{SR}_{1}$ & 20 \\
$\mathrm{SR}_{2}$ & 10 \\
$\mathrm{SR}_{3}$ & 10 \\
$\theta$ & 1 \\
$B$ & 12 \\
$\lambda$ & 2 \\
$C_{1}$ & 30 \\
$C_{2}$ & 50 \\
$C_{3}$ & 18 \\
$F$ & 10 \\
$Y_{1}$ & 20 \\
$Y_{2}$ & 25 \\
$\alpha$ & 0.5 \\
\hline
\end{tabular}

evolution point (weak control model, low-quality of service, and low frequency of use). In the case of scenario 3 , there is a stable evolution point (strong control model, high-quality service, and low frequency of use). Therefore, the government should strengthen regulation during the development of transport infrastructure to promote the provision of high-quality services by operational service providers, while providing users with a diversity of transport options.

\section{A BP Neural Network-Based Prediction Model for Sustainable Operation of Transportation Infrastructure}

In order to investigate whether the above results based on game theory are in line with reality and whether the conclusions can serve as a guide to reality, based on the simulation using MATLAB, the paper uses BP neural network to

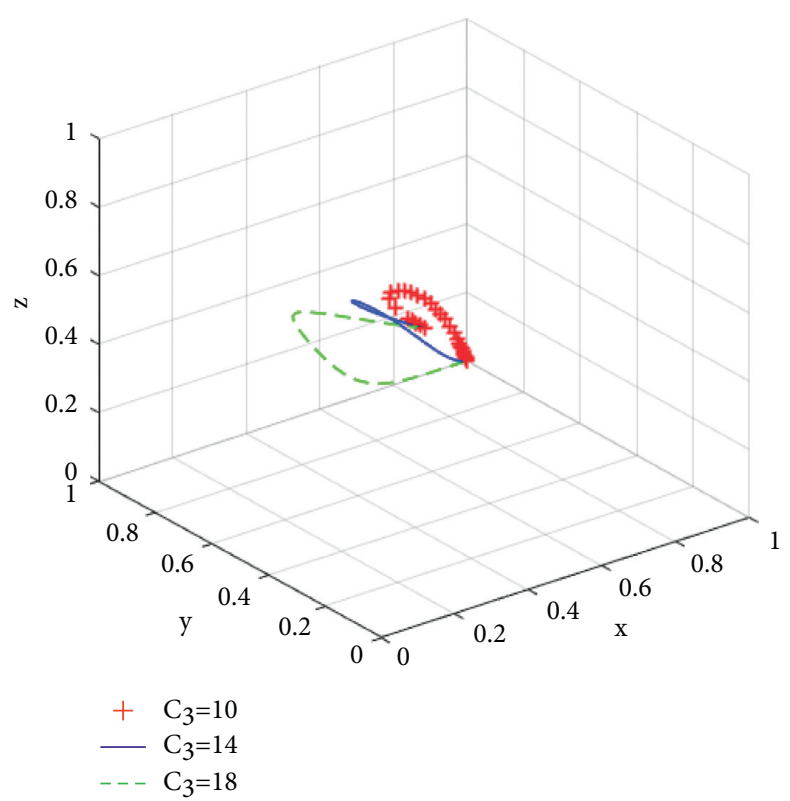

FIGURE 2: Impact of regulatory costs.

build a prediction model based on the results of game theory by collecting actual operation cases of transportation infrastructure across the country and investigates whether the conclusions obtained from the evolutionary game are correct and whether the results of the evolutionary game can be used to further guide the future construction. Based on the results of the game and the sensitivity of each parameter, the index parameters in the BP neural network construction process are further selected. The indicators selected in the model construction process are based on the indicators used in the evolutionary game process, including government subsidies, 


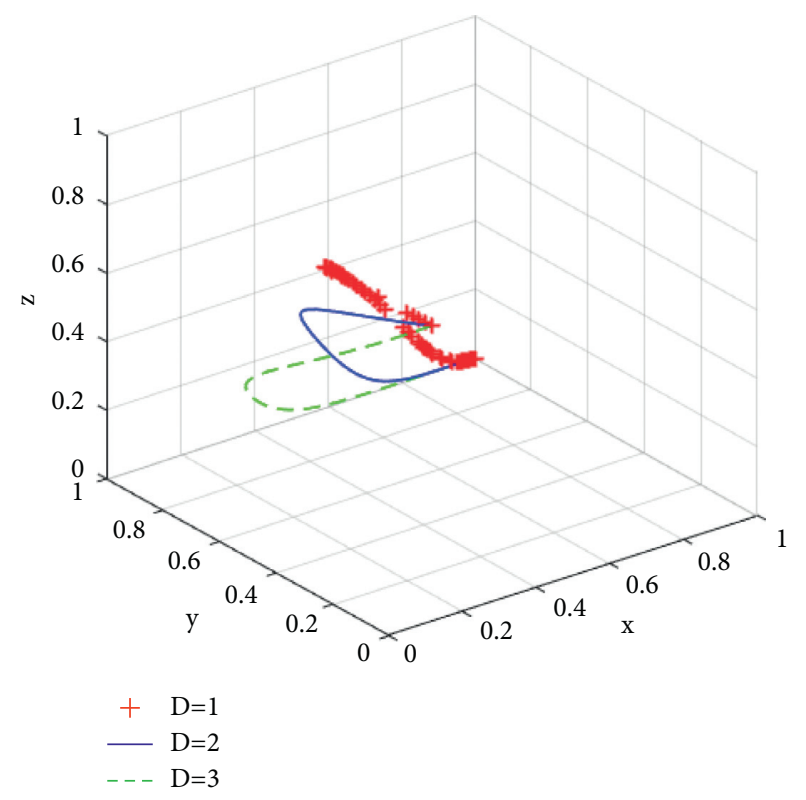

Figure 3: Impact of risk aversion factors.

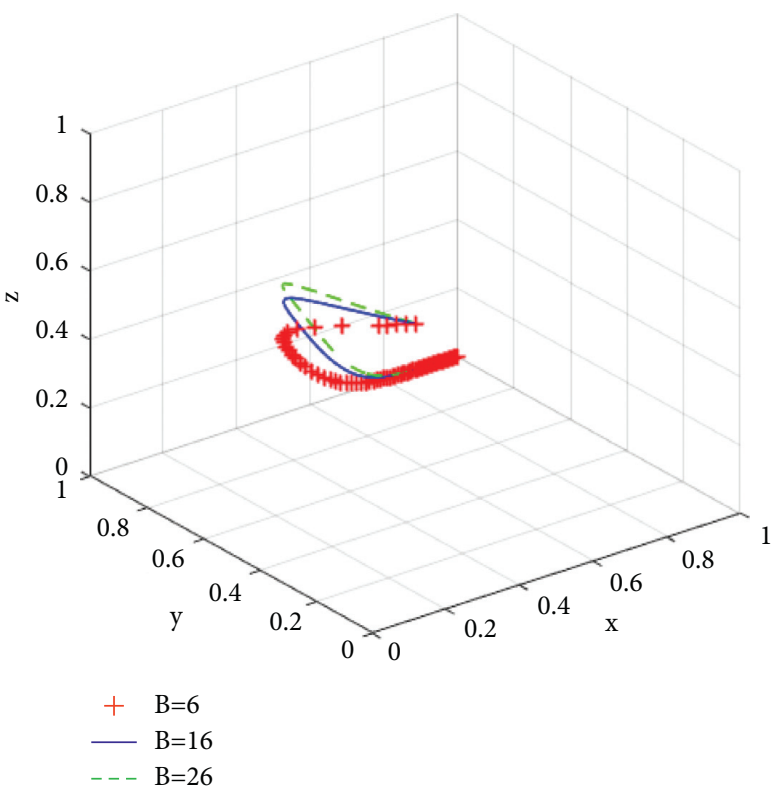

FIgURE 4: Impact of incentive grants.

government support, GDP, operating income, and other indicators, and supplemented with data indicators that reflect the scale of the project. The selected evaluation result is the final revenue of the project.

The relevant data of the paper are obtained from China National Statistical Yearbook, local economic operation reports, and other official public data.

The mean square error of the model is shown in Figure 8, and the model fit data are shown in Figure 9.

From the fitted data (Figure 9), it can be found that the outcome of the transportation infrastructure operation process can be predicted better using the indicators determined by the evolutionary game and sensitivity analysis of

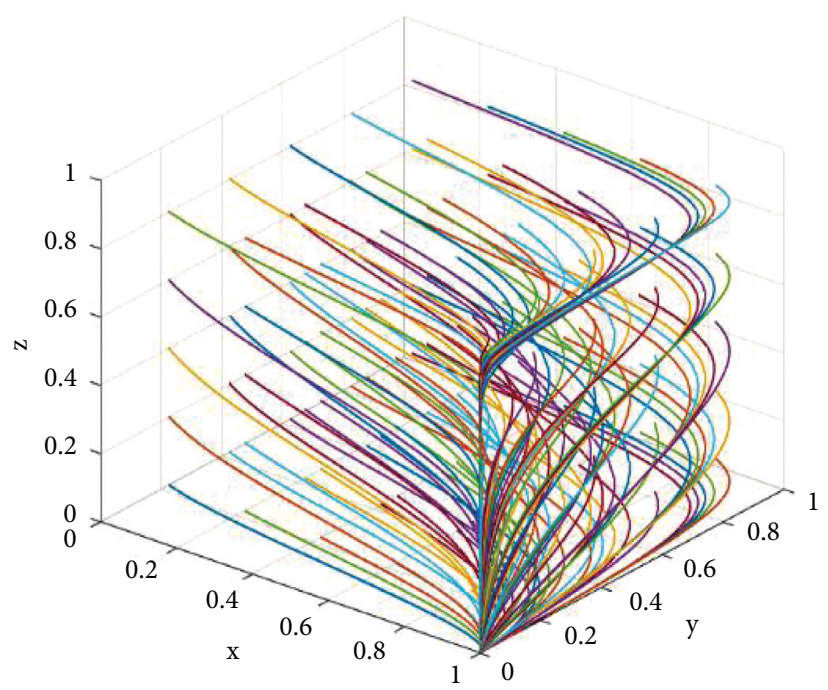

FIGURE 5: The results of 50 evolutions of situation 1 .

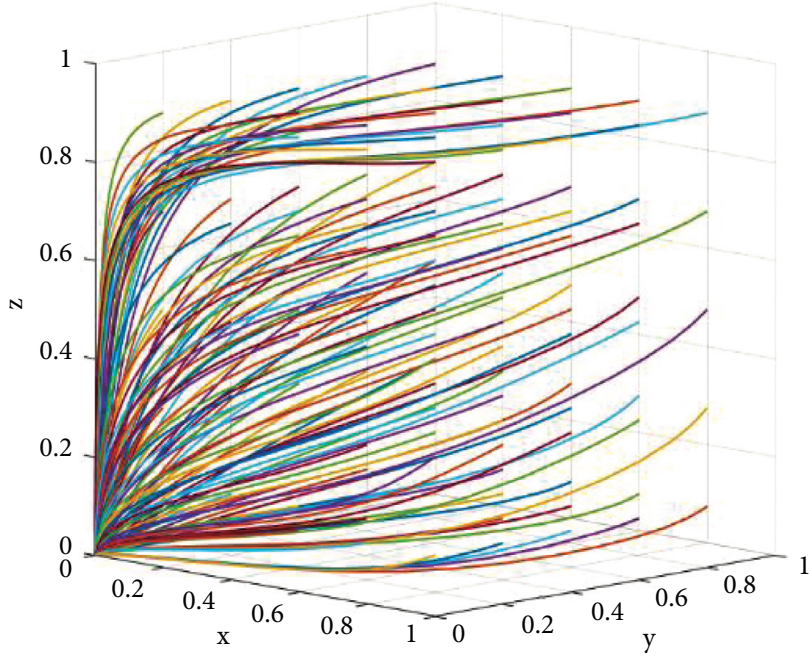

Figure 6: The results of 50 evolutions of situation 2.

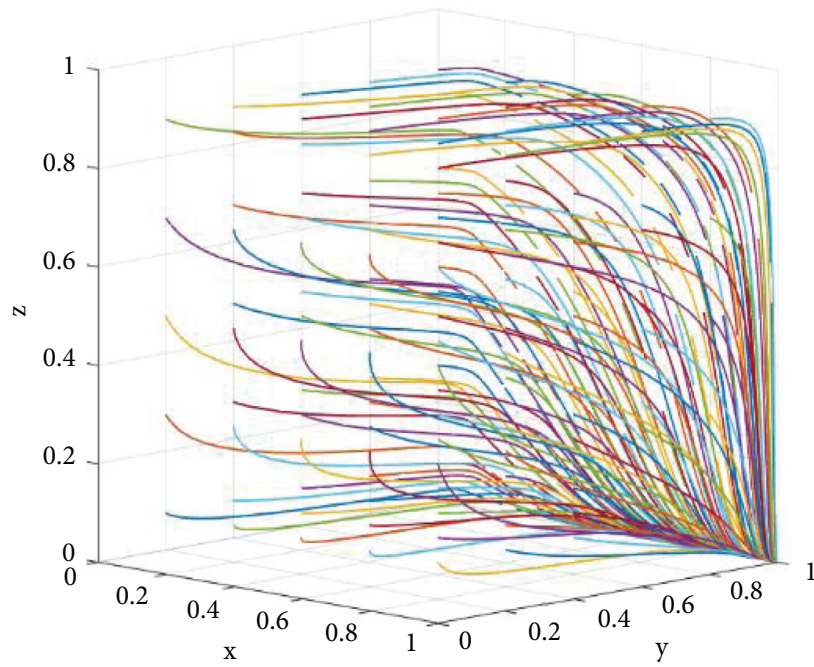

FIgURE 7: The results of 50 evolutions of situation 3 . 


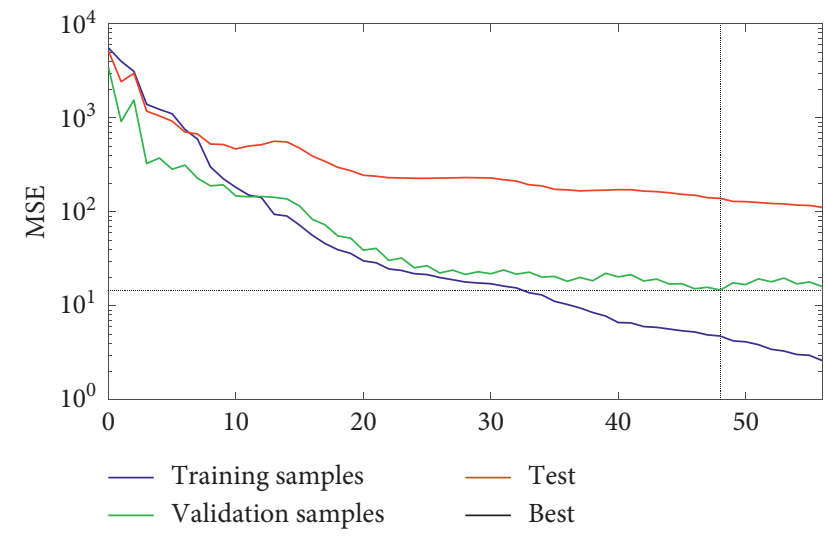

Figure 8: Mean square error.

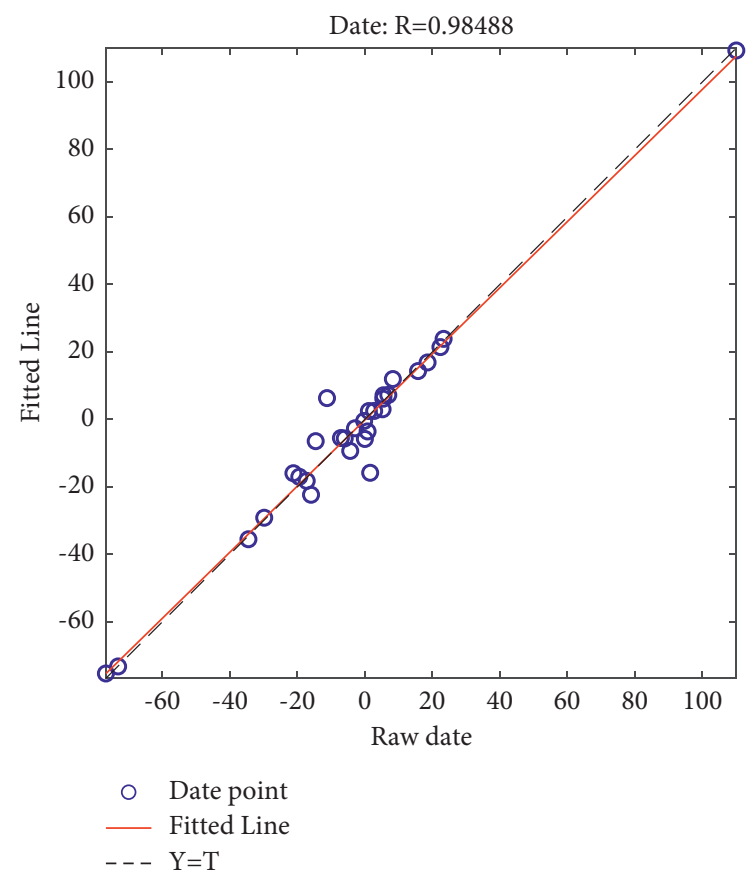

Figure 9: Sample regression plot.

indicators. The mean square error of the original data and the fitted data are small, and the BP neural network can be used to predict and analyze the operation process of the transportation infrastructure. At the same time, by adjusting the corresponding indexes in the prediction set according to the results of game theory analysis, we can find that the error of the model constructed by the BP neural network is smaller than that of the conclusion obtained by using the evolutionary game. Therefore, the combination of the deep learning method and game theory in the traditional operation process can better assist the government and the operator to predict and analyze the possible situation of the project. The use of the BP neural network can better predict the operation results of transportation infrastructure by external influencing factors; therefore, relevant deep learning algorithms can be added to analyze and evaluate the feasibility of the project in the process of relevant policy formulation and project evaluation.

\section{Conclusions}

This paper introduces prospect theory to the sustainable operation of transport infrastructure projects under government regulation, establishes an evolutionary game model with the participation of three parties: government, operator, and user, and analyzes it using relevant theories to obtain the following conclusions.

(1) The growth rate of the three parties in the game to participate in the construction of transport infrastructure by adopting positive strategies is not the same, and the size of the government's incentive for the construction operator to adopt positive strategies affects the government's willingness to participate. Excessive rewards are not only more burdensome for the government but also do not encourage operators to improve their service quality

(2) The government's willingness to participate is influenced by the cost of monitoring the low-quality of services provided by the operator. The government should dynamically adjust the level of supervision according to the operator's own positive level of operation and the actual operating results and control the supervision expenses while ensuring the operating results. Strong government control does not necessarily result in high-quality service from the service provider. In some cases, it has led to lowquality services being provided. Therefore, the interests of the operators need to be taken care of in the control process as well

(3) In all cases of evolutionary stability, users tend to choose low-frequency strategies, and the government should provide users with diverse transport options to meet their needs. The government should provide users with a wide range of transport options to meet their needs, and in the construction of the city, it should balance the development of each region as far as possible and build up distinctive industries in each region to avoid the emergence of "sleeping cities"

(4) In many cases, operators evolve a balanced strategy of "choosing low-quality services" in order to maximize their own profitability. This is a reflection of the fact that operators are currently rewarded for providing high-quality services and are not sufficiently penalized for providing low-quality services, resulting in operators choosing to adopt low-quality services in order to avoid loss of profit during the operation process, which is not conducive to good project operation

(5) In the process of research for infrastructure operations, the use of deep learning algorithms can better simplify the original research process on the basis of complex calculations to obtain similar conclusions and at the same time can realize the prediction of the future based on the underlying data. Therefore, in the process of future research and use, we can try to 
combine deep learning algorithms with traditional analysis and evaluation methods to further optimize the operation process

\section{Data Availability}

The raw data supporting the conclusions of this article will be made available by the authors, without undue reservation.

\section{Conflicts of Interest}

The authors declare that they have no conflicts of interest regarding this work.

\section{Acknowledgments}

This paper was funded by the National Natural Science Foundation of China (71771031).

\section{References}

[1] R. Gao, "Research on the success criteria of PPP projects," Management Modernization, vol. 38, no. 02, pp. 96-100, 2018.

[2] T. Besley and M. Ghatak, "Government versus private ownership of public goods," Quarterly Journal of Economics, vol. 116, no. 4, pp. 1343-1372, 2001.

[3] C. Xu, "Comparative study on subsidy models of government and social capital cooperation (PPP) projects--based on the practice of urban rail transit PPP projects," Macroeconomic Research, vol. 94, no. 05, 106+165 pages, 2018.

[4] F. Medda, "A game theory approach for the allocation of risks in transport public private partnerships," International Journal of Project Management, vol. 25, no. 3, pp. 213-218, 2007.

[5] P. Xia and F. Gao, "Research on social regulation strategy of infrastructure operation based on credit game," Management Modernization, vol. 38, no. 05, pp. 66-71, 2018.

[6] C. Jiang, "Regulatory mechanism of PPP mode operation from the perspective of game theory," Gansu Finance, vol. 20, no. 01, pp. 58-62, 2017.

[7] W. He, "Research on government supervision of urban infrastructure under PPP mode," China Business Theory, vol. 26, no. 33, pp. 152-154, 2017.

[8] N. M. El-Gohary, H. Osman, and E. E. D. Tamer, "Stakeholder management for public private partnerships," International Journal of Project Management, vol. 24, no. 7, 2006.

[9] Y. Wu, "Research on the introduction of PPP model for infrastructure stock assets," Macroeconomic Research, vol. 40, no. 02, pp. 84-91, 2020.

[10] W. Sun, "Empirical laws and strategy optimization of PPP mode financing for infrastructure construction--an analysis based on two typical cases," Economic Vertical, vol. 41, no. 07, pp. 120-128, 2019.

[11] Y. Yang and M. Chen, "On the structural model and tax policy of REITs--Taking infrastructure public REITs as an example," Finance and Accounting Monthly, vol. 29, no. 13, pp. 133-141, 2021.

[12] Z. Zhang, M. Jia, and W. D. Fang, "The optimal control allocation of PPP from the perspective of incomplete contract and relationship contract," Foreign Economics and Management, vol. 24, no. 08, 29+44 pages, 2007.

[13] E. T. Ma and X. Li, "Research on the cooperative relationship between project participants under PPP model--an analytical framework based on social network theory," Finance and Trade Economics, vol. 38, no. 07, pp. 49-63+77, 2017.

[14] Y. He and H. Xiong, "Research on government supervision of PPP mode from the perspective of game theory," Journal of Engineering Management, vol. 32, no. 06, pp. 64-68, 2018.

[15] W. Liu, J. N. Lv, and Q. Zou, "Decision model for concession period of transportation BOT projects under revenue uncertainty," Systems Engineering, vol. 30, no. 12, pp. 51-56, 2012.

[16] B. Liu and W. Liu, "Game analysis of private supply mechanism of quasi-public goods: the case of transportation infrastructure investment in China," China Soft Science, vol. 22, no. 08, pp. 145-151, 2007.

[17] Z. G. Shao, C. Han, L. Meng, and Q. Wu, "A logistic-based model for the evolution of regional transportation infrastructure ecosystem," Systems Engineering Theory and Practice, vol. 38, no. 11, pp. 2918-2928, 2018.

[18] D. Yanchao, Z. Hengyu, Y. Yongbo, and L. Xiaoxue, "Explore knowledge-sharing strategy and evolutionary mechanism for integrated project team based on evolutionary game model," Advances in Civil Engineering, vol. 2019, Article ID 4365358, 23 pages, 2019.

[19] H. A. Mesa, K. R. Molenaar, and L. F. Alarcón, "Exploring performance of the integrated project delivery process on complex building projects," International Journal of Project Management, vol. 34, no. 7, pp. 1089-1101, 2016.

[20] D. R. Hale, P. P. Shrestha, G. E. Gibson, and G. C. Migliaccio, "Empirical comparison of design/build and design/bid/build project delivery methods," Journal of Construction Engineering and Management, vol. 135, no. 7, pp. 579-587, 2009.

[21] Q. Shi, J. Zhu, L. Qian, and P. D. Lellis, "Cooperative Evolutionary Game and Applications in Construction Supplier Tendency," Complexity, vol. 2018, Article ID 8401813, 13 pages, 2018.

[22] T. Feng, S. Tai, C. Sun, and Q. Man, "Study on cooperative mechanism of prefabricated producers based on evolutionary game theory," Mathematical Problems in Engineering, vol. 2017, Article ID 1676045, 6 pages, 2017.

[23] D. Friedman, "Evolutionary games in economics," Econometrica, vol. 59, no. 3, pp. 637-666, 1991.

[24] J. Liu, R. Gao, C. Y. J. Cheah, and J. Luo, "Evolutionary game of investors' opportunistic behaviour during the operational period in PPP projects," Construction Management \& Economics, vol. 35, no. 3, pp. 137-153, 2017.

[25] Y. Lei, "A tripartite evolutionary game analysis of the Coconstruction of an international transport corridor," Discrete Dynamics in Nature and Society, vol. 12, no. 2021, pp. 1-11, 2021.

[26] X. Sun and Y. Lei, "Research on financial early warning of mining listed companies based on BP neural network model," Resources Policy, vol. 73, Article ID 102223, 2021.

[27] L. Bai, K. Zheng, Z. Wang, and J. Liu, "Service provider portfolio selection for project management using a BP neural network," Annals of Operations Research, pp. 1-22, 2021. 